\title{
Caracterización de pacientes con accidente cerebrovascular ingresados en un hospital de baja complejidad en Chile
}

\author{
Characterization of patients with cerebrovascular accident admitted to a primary care hospital in Chile
}

Jorge Sepúlveda-Contreras ${ }^{1 *}$ orcid.org/0000-0002-7060-2475

1. Hospital San Luis de Buin-Paine, Chile

\section{Resumen}

Introducción: El Accidente cerebrovascular se constituye como un problema crítico de salud pública, siendo sus mayores factores de riesgo la hipertensión, el consumo de tabaco, la diabetes, entre otros. Objetivo: Caracterizar a los usuarios que ingresan con diagnóstico de Accidente cerebrovascular al Hospital San Luis de Buin-Paine, hospital de baja complejidad perteneciente al Servicio de Salud Metropolitano Sur de Chile. Materiales y métodos: Se realizó una investigación observacional, descriptiva, transversal con aquellos pacientes que ingresaron a servicios de hospitalizados y a la unidad de rehabilitación con diagnóstico de Accidente cerebrovascular isquémico o hemorrágico. Con la muestra $(n=135)$ se realizó una revisión de cada ficha electrónica. El procesamiento estadístico de los datos fue mediante frecuencias absolutas y porcentajes. Resultados: Predominó el ictus isquémico en el 85,92\%, con un fuerte incremente en la población sobre 51 años. Los factores de riesgos más frecuentes fueron hipertensión arterial y diabetes mellitus. La profesión u oficio que más se repitió correspondió a Jubilado (27,4\%). Conclusiones: Se evidenciaron resultados similares en comparación a otras investigaciones afines al tema. La prevención de las enfermedades cerebrovasculares está asociada al mantenimiento de hábitos y estilos de vida saludables para prevenir la aparición de los factores modificables de riesgo.

Palabras clave: Accidente cerebrovascular; factores de riesgo; epidemiología. (Fuente: DeCS, Bireme).

\begin{abstract}
Introduction: Cerebrovascular accidents constitute a critical public health problem, whose major risk factors are hypertension, tobacco use and diabetes, among others. Objective: To characterize patients admitted with a diagnosis of cerebrovascular accident to the San Luis de Buin-Paine Hospital, which is a primary care institution belonging to the Southern Metropolitan Health Service of Chile. Materials and methods: An observational, descriptive and cross-sectional research was carried out with patients who were hospitalized in the rehabilitation unit with a diagnosis of ischemic or hemorrhagic cerebrovascular accident. A review of each electronic record was carried out with a sample containing 135 patients. Absolute frequencies and percentages were used for statistical processing of the data. Results: Ischemic stroke was observed in $85.92 \%$ of patients, with a significant percentage increase in the population over 51 years of age. The most frequent risk factors were high blood pressure and diabetes mellitus. The most common profession or trade was retiree (27.4\%). Conclusions: This work found similar results to those reported by previous studies. Prevention of cerebrovascular diseases is associated with the maintenance of healthy habits and lifestyles that prevent the appearance of modifiable risk factors.
\end{abstract}

Keywords: Stroke; risk factors; epidemiology. (Source: DeCS, Bireme).

\author{
*Autor de correspondencia \\ Jorge Sepúlveda-Contreras \\ e-mail: fonoaudiologo.jorgesepulveda@gmail.com
}




\section{Introducción}

El Accidente cerebrovascular (ACV) ocurre cuando se interrumpe el suministro de sangre al cerebro o cuando existe un sangrado en él(1), siendo definido por la Organización Mundial de la Salud (OMS) como el rápido desarrollo de signos focales o globales de compromiso de la función cerebral, con una duración de síntomas entre las 24 horas o más, o que lleven a la muerte, sin otra causa que el origen vascular(2). Los tipos más habituales son el isquémico y el hemorrágico. El ACV isquémico es el más frecuente, y se produce por un bloqueo del flujo normal de sangre hacia el cerebro. El ACV hemorrágico es menos frecuente pero más letal, y se produce cuando se rompe un vaso sanguíneo en el cerebro originando un derrame ${ }^{(1)}$.

Los factores de riesgo a la hora de provocar un ACV pueden ser clasificados como no modificables y modificables. Entre los no modificables se encuentra la edad, el sexo, y el tener antecedentes de familiares directos con ACV; de manera que el riesgo aumenta con la edad, ser de sexo masculino y tener familiares directos que hayan padecido un ataque cerebral ${ }^{(1)}$. En relación a los factores de riesgos modificables, existe abundante evidencia de asociación con la presión arterial elevada, el consumo de tabaco, la diabetes, altos niveles de colesterol, la obesidad y el antecedente de fibrilación auricular(1,3-6); entre todos ellos, se destaca la hipertensión como el factor de riesgo más común (4-6).

Según datos del Ministerio de Salud de Chile (MINSAL), en el año 2011 en la región metropolitana se presentaron 3.236 defunciones, producto de enfermedades cerebrovasculares, siendo la primera causa de muerte y representando el 9\% de ellas durante el año 2010(7). En Estados Unidos (EEUU), el ACV fue la quinta causa de muerte con una prevalencia general del $2,6 \%$ en los mayores de 20 años entre los años 2009 y 2012, siendo a su vez el ACV isquémico el más prevalente con un $85 \%$ de los $\operatorname{casos}^{(4)}$.

El Proyecto Investigación de Stroke, Iquique Stroke Study (PISCIS) en Chile, único estudio amplio realizado hasta la fecha, reportó que el $44 \%$ de los sujetos identificados con el primer ACV fueron mujeres y el 56\% fueron hombres, con edades medias de 68,5 años y 61,2 años, respectivamente ${ }^{(8)}$. Los factores de riesgo modificables presentaron una prevalencia de: hipertensión $60 \%$, diabetes mellitus
$21 \%$, dislipidemia $14 \%$, enfermedades coronarias $24 \%$, consumo de cigarros $15 \%$, y consumo de alcohol $13 \%{ }^{(8)}$. Dicha incidencia y prevalencia es constante en diversos estudios relacionados al tema ${ }^{(4,6,69}$.

Frente a lo expuesto anteriormente surgieron los interrogantes: ¿Los factores de riesgo modificables observados en estudios anteriores se encontrarán en igual medida al investigar a otros grupos con ACV? ¿Se apreciarán diferencias entre los casos de ACV según la zona de procedencia, ya sea rural o urbano?

Para estas inquietudes se planteó el objetivo de caracterizar a los usuarios que ingresan con diagnóstico de Accidente cerebrovascular al Hospital San Luis de Buin-Paine, institución de baja complejidad perteneciente al Servicio de Salud Metropolitano Sur de Chile. Con los datos recabados se pretende discutir sobre los principales factores de riesgo modificables, con una mirada orientada finalmente en su prevención.

\section{Materiales y métodos}

Se realizó una investigación observacional, descriptiva, transversal con aquellos pacientes que ingresaron a servicios de hospitalizados y a la unidad de rehabilitación del Hospital San Luis de Buin Paine (HSLB), con diagnóstico de ACV isquémico o hemorrágico, y que hayan sido evaluados por el equipo de neurorehabilitación del HSLB entre el periodo de septiembre de 2018 a septiembre de 2019. Los diagnósticos de ACV fueron confirmados mediante estudios de tomografía axial computarizada (TAC) y/o resonancia magnética (RM), validados por un profesional del hospital de mayor complejidad de procedencia. La recopilación de antecedentes para realizar la caracterización fue efectuada por el equipo de neurorehabilitación, compuesto por una kinesesióloga, una terapeuta ocupacional y un fonoaudiólogo.

Se aplicó una entrevista estándar orientada a obtener información sobre antecedentes personales, antecedentes mórbidos, lugar de residencia, ocupación, entre otras variables de utilidad terapéutica. Posteriormente, se corroboró los datos obtenidos con la información registrada en las fichas electrónicas de cada paciente, disponibles en la red informática del establecimiento. El procesamiento estadístico de los datos se realizó utilizando frecuencias absolutas $y$ porcentajes en la herramienta Excel de Microsoft. 


\section{Consideraciones éticas}

El estudio fue aprobado por el Comité Ético Científico del Servicio de Salud Metropolitano Sur el 27 de diciembre de 2019, sin la necesidad de requerir consentimiento informado por parte de los pacientes ingresados, debido a las características del estudio y a que se mantuvo el anonimato y la confidencialidad de los datos recolectados.

\section{Resultados}

Durante el periodo septiembre 2018 a septiembre 2019, ingresaron al Hospital San Luis de Buin - Paine 135 usuarios con el diagnóstico confirmado de ACV. De ellos, 114 pacientes ingresaron a servicios de hospitalización y 21 directamente a la Unidad de Rehabilitación provenientes del Hospital Barros Luco Trudeau (hospital base de alta complejidad, perteneciente al Servicio de Salud Metropolitano Sur), 19 de Atención Primaria de Salud, uno de otro recinto hospitalario y uno de la Región de la Araucanía, Chile.

El promedio de edad de los sujetos registrados fue de 70,47 años, con mayor prevalencia el sexo masculino (54,07\%). Entre las profesiones $\mathrm{y} / \mathrm{u}$ oficios desempeñados por los pacientes, se encontró que el $27,4 \%$ eran jubilados, el $23,7 \%$ amas de casa, el $11,1 \%$ agricultores, y el $10,3 \%$ choferes de locomoción colectivo o conductores.

En relación al lugar de residencia de los usuarios el $58 \%(n=79)$ proviene de la comuna de Buin, a la cual pertenecen las localidades de: Maipo (18,5\%), Buin centro $(14,1 \%)$, Valdivia de Paine $(7,4 \%)$, Linderos $(5,9 \%)$ y Viluco $(5,2 \%)$. El $41 \%$ provenían de la comuna de Paine $(n=55)$ y sus localidades de Paine centro $(12,6 \%)$ y Águila sur $(11,9 \%)$. Un usuario ingresó proveniente de otro recinto hospitalario, por lo que su lugar de residencia no corresponde a los sectores mencionados $(n=1)$.

El ictus que predominó fue el isquémico en un $85,92 \%$ de los pacientes, observando un fuerte incremento de casos desde el grupo etario de 51-60 años (Tabla 1). En cuanto al tipo de ictus por sexo, prevaleció en el sexo masculino con ambos tipos de ACV en un 54,07\% (Tabla 1). En cuanto a los antecedentes mórbidos asociados al ACV, predominó la hipertensión en ambos tipos de enfermedad cerebrovascular, seguidos de la diabetes mellitus y de presencia de enfermedad coronaria. La dislipidemia y el consumo de tabaco tuvieron un porcentaje importante en ambos tipos de ictus (Tabla 2).

Tabla 1. Predominio de tipo de ACV por rango de edad o sexo

\begin{tabular}{|c|c|c|c|c|c|c|}
\hline \multirow{2}{*}{ Característica } & \multicolumn{2}{|c|}{ Isquémico } & \multicolumn{2}{|c|}{ Hemorrágico } & \multicolumn{2}{|c|}{ Total } \\
\hline & $\mathrm{n}$ & $\%$ & $\mathbf{N}$ & $\%$ & $\mathbf{n}$ & $\%$ \\
\hline \multicolumn{7}{|l|}{ Edad (años) } \\
\hline $30-40$ & 4 & 2,96 & 0 & 0 & 4 & 2,96 \\
\hline $41-50$ & 2 & 1,48 & 4 & 2,96 & 6 & 4,44 \\
\hline $51-60$ & 27 & 20 & 3 & 2,22 & 30 & 22,22 \\
\hline $61-70$ & 24 & 17,77 & 6 & 4,44 & 30 & 22,22 \\
\hline $71-80$ & 31 & 22,96 & 1 & 0,74 & 32 & 23,70 \\
\hline 81 y más & 28 & 20,74 & 5 & 3,70 & 33 & 24,44 \\
\hline Total & 116 & 85,92 & 19 & 14,07 & 135 & 100 \\
\hline \multicolumn{7}{|l|}{ Sexo } \\
\hline Masculino & 63 & 46,66 & 10 & 7,40 & 73 & 54,07 \\
\hline Femenino & 53 & 39,25 & 9 & 6,66 & 62 & 45,92 \\
\hline Total & 116 & 85,92 & 19 & 14,0 & 135 & 100 \\
\hline
\end{tabular}

Tabla 2. Predominio de factores de riesgo modificables por tipo de ACV

\begin{tabular}{|c|c|c|c|c|c|}
\hline \multirow{2}{*}{ Factor de riesgo } & \multicolumn{2}{|c|}{ Isquémico (=116) } & \multicolumn{2}{|c|}{ Hemorrágico (=19) } & \multirow{2}{*}{\begin{tabular}{|c|} 
Total \\
$\%$ \\
\end{tabular}} \\
\hline & $\mathbf{n}$ & $\%$ & $\mathbf{n}$ & $\%$ & \\
\hline Hipertensión arterial & 92 & 79,31 & 16 & 84,21 & 80 \\
\hline Diabetes mellitus & 45 & 38,79 & 6 & 31,57 & 37,77 \\
\hline Dislipidemia & 22 & 18,96 & 6 & 31,57 & 20,74 \\
\hline Enfermedad coronaria & 37 & 31,89 & 6 & 31,57 & 31,85 \\
\hline Consumo de tabaco & 31 & 26,72 & 5 & 26,31 & 26,66 \\
\hline Consumo de alcohol & 3 & 2,58 & 3 & 15,78 & 4,44 \\
\hline Consumo de drogas & 0 & 0 & 1 & 5,26 & 0,74 \\
\hline
\end{tabular}




\section{Discusión}

El presente estudio muestra una mayor prevalencia de la enfermedad en la población masculina, con un promedio de edad de 70 años. Los resultados son similares a los observados en diversos estudios nacionales e internacionales $(4,6,8,9)$, donde la incidencia de ACV se incrementa notoriamente con la edad, y a partir de los 55 años las probabilidades de sufrirlo se duplican(10).

En relación a los factores de riesgo modificables, fueron similares a los encontrados en otros trabajos. La hipertensión arterial fue el factor que más predominó en ambos tipos de accidentes vasculares, constituye la principal causa para enfermedades cerebrovasculares, siendo su relación con la enfermedad cerebrovascular una de las más documentadas $(4,5,10,11)$.

La diabetes mellitus, las enfermedades coronarias y la dislipidemia, también estuvieron entre las primeras condiciones de riesgo para la enfermedad cerebrovascular. La diabetes mellitus es altamente prevalente en pacientes con accidente cerebrovascular ${ }^{(4)}$ y en aquellos que la padecen por más de 10 años, el riesgo de sufrir un ACV se triplica(12). Los datos observados en esta investigación fueron mayores a los evidenciados en el estudio PISCIS $(21 \%, 11 \%$, y $14 \%$ respectivamente del estudio PISCIS(8), versus el 37\%, 31\% y 20\%, de la presente investigación).

En cuanto al consumo de drogas y alcohol se encuentran por debajo de lo evidenciado en el estudio PISCIS(8); por el contrario en relación al consumo de tabaco se presentaron resultados más altos al realizar la comparación $26,6 \%$ y 15\%, respectivamente). Fumar se ha asociado fuertemente con un mayor riesgo de sufrir un accidente cerebrovascular, de manera que los fumadores tienen un doble riesgo, independientemente de la existencia de otros factores asociados $(4,5,13)$.

A diferencia de otros estudios, esta investigación consideró los oficios y las comunas de residencia de los usuarios ingresados; se observó un gran porcentaje de pacientes con oficios del sector agrícola y de conducción de locomoción colectiva y un $27,4 \%$, se encontraban jubilados. Es importante destacar que muchos usuarios residían en los sectores rurales de la zona, lo cual podría influir en una baja adherencia a los controles preventivos en atención primaria de salud. No obstante, esta hipótesis requiere de futuras investigaciones, donde se establezcan posibles relaciones entre edad, ubicación del domicilio y adherencia a controles preventivos, como lo concluido por Varleta et al.,(14) con pacientes mayores de 64 años, edad que en Chile correspondería a la población jubilada.

Las enfermedades cerebrovasculares son un grave problema de salud, y las acciones de promoción y prevención desempeñan un importante papel(11), dado que buscan disminuir su incidencia mediante la modificación dirigida de un solo factor de riesgo, o un grupo de múltiples factores de riesgo en la población, comunidad o individuo ${ }^{(15)}$.

Los resultados de esta investigación pueden ser clave a la hora de generar acciones preventivas específicas para la población estudiada. Estas acciones deben centrarse en el mantenimiento de hábitos y estilos de vida saludables, para evitar la aparición de los factores de riesgos y controlar o eliminar los existentes $\left({ }^{(9)}\right.$. El ACV no es producto del azar, sino que parte de un proceso de desarmonía gestado a lo largo de la vida(13), y la mayoría de los factores de riesgo son modificables, por ello, es importante proporcionar una educación más rigurosa a aquellos que están en alto riesgo(4).

\section{Conclusiones}

La información recolectada será clave para generar nuevas acciones preventivas encaminadas al mantenimiento de hábitos y estilos de vida saludables para prevenir la aparición de los factores modificables de riesgo; como también para realizar futuras investigaciones orientadas a establecer relaciones de adherencia a tratamientos farmacológicos en determinados grupos de riesgo.

Los resultados obtenidos en la presente investigación deben ser interpretados atendiendo sus limitaciones, como el hecho de no contar con un registro ordenado y protocolizado.

\section{Agradecimientos}

A las profesionales Elizabeth Herrera y Daniela Ramos, por su ayuda y apoyo incondicional.

\section{Conflicto de intereses}

No se declaran conflictos de interés en la presente investigación. 


\section{Referencias}

1. MINSAL. Plan de acción [Internet]. 2017. Disponible en https://www.minsal.cl/ataque_cerebral/

2. Goldstein M, Barnett H, Orgogozo JM, Sartorius N, Symon L, Vereshchagin NV, et al. Recommendations on stroke prevention, diagnosis, and therapy. Stroke. 1989 Oct;20(10):1407-8. doi:10.1161/01.str.20.10.1407

3. Mozaffarian D, Benjamin EJ, Go AS, Arnett DK, Blaha MJ, Cushman M, et al. Heart disease and stroke statistics-2016 update a report from the American Heart Association. Circulation. 2016 Jan 26;133(4):e38-48. doi:10.1161/CIR.0000000000000350.

4. Guzik A, Bushnell C. Stroke Epidemiology and Risk Factor Management. Contin Lifelong Learn Neurol. 2017 Feb;23(1):15-39. doi:10.1212/con.0000000000000416

5. Sherzai AZ, Elkind MSV. Advances in stroke prevention. Ann N Y Acad Sci. 2015 Mar;1338(1):1-15. doi:10.1111/nyas.12723

6. Fekadu G, Chelkeba L, Kebede A. Risk factors, clinical presentations and predictors of stroke among adult patients admitted to stroke unit of Jimma university medical center, south west Ethiopia: prospective observational study. BMC Neurol. 2019 Dec 7;19(1). doi: 10.1186/s12883-019-14090 .

7. MINSAL. Departamento de Estadísticas e Información en Salud [Internet]. 2016. Disponible en: http://www.deis.cl/defunciones-y-mortalidad-por-causas/.

8. Lavados PM, Sacks C, Prina L, Escobar A, Tossi C, Araya F, et al. Incidence, 30-day case-fatality rate, and prognosis of stroke in Iquique, Chile: A 2-year community-based prospective study (PISCIS project). Lancet. 2005 Jun;365(9478):2206-15. 6736(05)66779-7
9. Piloto González R, Herrera Miranda GL, Ramos Aguila Y de la C, Mujica González DB, Gutiérrez Pérez M. Caracterización clínica-epidemiológica de la enfermedad cerebrovascular en el adulto mayor. Revista de Ciencias Médicas de Pinar del Río. 2015;19(6). Disponible en: http://scielo.sld.cu/scielo.php?script=sci_arttext\&pid=S156 1-31942015000600005\&lng=es.

10. Berenguer Guarnaluses L, Pérez Ramos A. Factores de riesgo de los accidentes cerebrovasculares durante un bienio. Medisan. 2016;20(5):621-629. Disponible en: http://scielo.sld.cu/scielo.php?script=sci_arttext\&pid=S102 9-30192016000500005\&lng=es.

11. Ramos Fernández 0 , Menéndez Rodríguez JC, Puentes Colombé M, Benítez Pozo OL, Sánchez Hernández E. Factores de riesgo de enfermedades cerebrovasculares en pacientes atendidos en unidad de cuidados intensivos municipal. Rev Ciencias Médicas Pinar del Río. 2020;24(2):171-7. Disponible en: http://revcmpinar.sld.cu/index.php/publicaciones/article/ view/4190

12. Ederle J, Brown MM. Stroke prevention, Herz. Treasure Island: StatPearls. 2008;33:518-523. Disponible en: https://www.ncbi.nlm.nih.gov/books/NBK470234/

13. Ruiz-Mejía AF, Pérez-Romero GE, Ángel-Macías MA. Stroke: Pathophysiology from the biomedical system perspective and its equivalent in the traditional Chinese medicine. Rev Fac Med. 2017 Jan 1;65(1):137-44. http://dx.doi.org/10.15446/revfacmed.v65n1.57508

14. Varleta P, Akel C, Acevedo M, Salinas C, Pino J, Opazo V, et al. Assessment of adherence to antihypertensive therapy. Rev Med Chil. 2015 May;143(5):569-76. http://dx.doi.org/10.4067/S0034-98872015000500003

15. Boehme AK, Esenwa C, Elkind MSV. Stroke Risk Factors, Genetics, and Prevention. Circ Res. 2017 Feb 3;120(3):47295. doi:10.1161/CIRCRESAHA.116.308398 